\title{
RICH SNIPPETS: INFORMACIÓN SEMÁNTICA PARA LA MEJORA DE LA IDENTIDAD DIGITAL Y EL SEO
}

\section{Cristòfol Rovira, Lluís Codina y Ricard Monistrol}
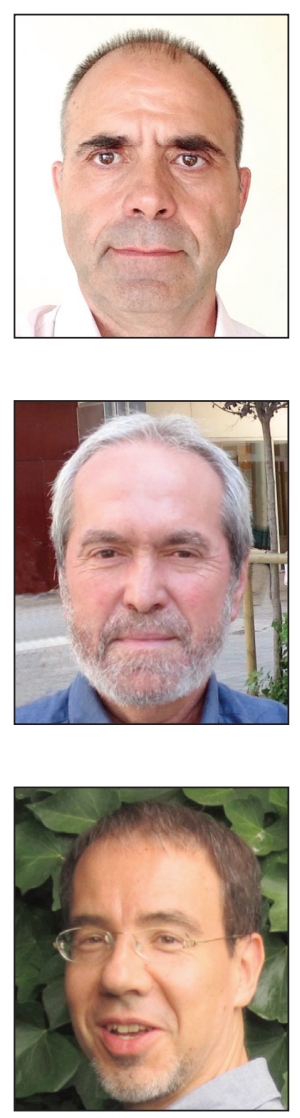

Cristòfol Rovira es profesor titular de la Universitat Pompeu Fabra (UPF). Imparte docencia en los grados de periodismo y de publicidad y relaciones públicas y en el Master oficial en gestión de contenidos digitales (Universidad de Barcelona \& UPF). Director del Master en documentación digital $(U P F)$ y del Master en Buscadores (UPF). Es investigador del grupo DigiDoc de la UPF y director de la revista académica Hipertext.net.

http://orcid.org/0000-0002-6463-3216

cristofol.rovira@upf.edu

Lluís Codina, profesor titular de universidad, imparte docencia en los estudios de periodismo y en la Facultad de Comunicación Audiovisual de la Universitat Pompeu Fabra (UPF) de Barcelona. Es el investigador principal del grupo de investigación DigiDoc de la misma universidad. Participa en el Master interuniversitario UB/UPF en gestión de contenidos digitales, en el programa de doctorado del Departamento de Periodismo y de Comunicación Audiovisual y es co-director del Master online de documentación digital.

http://orcid.org/0000-0001-7020-1631

Iluis.codina@upf.edu

Ricard Monistrol es consultor e investigador académico, actualmente finaliza su tesis doctoral en la Facultad de Biblioteconomía y Documentación de la Universitat de Barcelona (UB), posee el DEA en comunicación social por la Universitat Pompeu Fabra (UPF) y es licenciado en periodismo por la Universitat Autónoma de Barcelona (UAB). Realiza tareas de docencia en el IDEC y en el Departamento de Comunicación de la UPF y colabora con el grupo de investigación DigiDoc. Es el editor y director del anuario DifuCom, publicación académica y científica sobre patrimonio y TIC.

http://orcid.org/0000-0001-5459-4615

info@ricardmonistrol.eu

Universitat Pompeu Fabra Departamento de Comunicación, Grupo de investigación DigiDoc Roc Boronat, 138. 08018 Barcelona, España

\section{Resumen}

Se analizan y describen los rich snippets (descripciones enriquecidas) como un claro ejemplo de la web semántica real donde la metainformación se está aplicando para la mejora de los listados de resultados de los buscadores. Se describen los distintos tipos que admite Google y se explica cómo implementar los rich snippets de personas dada su importancia para la mejora de la identidad digital y su influencia en el posicionamiento web por medio del Author rank.

\section{Palabras clave}

Rich snippets, Microformatos, Microdatos, Metadatos, SEO, Posicionamiento, Web semántica, Author rank, Google.

Title: Rich snippets: Semantic information to improve digital identity and SEO

\begin{abstract}
The article analyzes and describes rich snippets as a clear example of the real semantic web where metadata is being applied to improve the search engine results pages (serps). We describe the different types of Google rich snippets and explain how to implement people snippets due to their importance for the improvement of digital identity and their impact on the SEO by influencing the Author rank.
\end{abstract}

\section{Keywords}

Rich snippets, Microformats, Microdata, Metadata, SEO, Search engine optimization, Semantic web, Author rank, Google. 
Rovira, Cristòfol; Codina, Lluís; Monistrol, Ricard (2013). "Rich snippets: información semántica para la mejora de la identidad digital y el SEO". El profesional de la información, noviembre-diciembre, v. 22, n. 6, pp. 554-561.

http://dx.doi.org/10.3145/epi.2013.nov.08

\section{Rich snippets y microdatos}

Nuestra identidad digital es un activo en sí mismo ya que nos aporta credibilidad y visibilidad. Además es cada vez más importante para el SEO (optimización de los resultados en los buscadores) ya que Google ha ido modificando sus algoritmos de ordenación de la lista de resultados en una doble dirección:

- otorga un peso mayor a los factores de credibilidad de un sitio, tales como una autoría bien determinada;

- innova continuamente en las formas de interpretar el contenido web mediante metadatos, con el fin de presentar páginas de resultados más informativas y útiles.

Las páginas de resultados de los motores de búsqueda (search engine result pages, serp) están obligadas a ser un prodigio de usabilidad: deben presentar la máxima información posible legible de un solo golpe de vista, en un espacio a la vez muy limitado y sin que tal acumulación de información resulte agobiante para el usuario.

Con la unión de metadatos y un formato de codificación determinado, Google 'entiende' parte de la semántica de las páginas que indiza

Uno de las últimas innovaciones de Google son los rich snippets, término que podríamos traducir como "resúmenes o descripciones enriquecidas". En una serp convencional, el snippet habitual consta de un pequeño conjunto de datos, en muchas ocasiones poco significativo: un título, dos líneas de texto con un resumen del contenido de la página (el snippet propiamente) que con suerte han sido extraídas del metadato description, el url, y poco más. Un rich snippet contiene más información, como veremos después, y en cada caso proporciona datos muy precisos de acuerdo con el tipo de contenido.

La cuestión es que Google (y cualquier buscador en general) es incapaz de generar páginas con resultados enriquecidos si las páginas no contienen determinados metadatos codificados de un cierta manera a partir de los cuales el buscador es capaz de identificar la información de manera certera y presentarla de forma adecuada. Literalmente, con la unión de los metadatos y de un formato de codificación determinado, Google "entiende" parte de la semántica de las páginas que indiza.

Por eso el conocimiento de esta clase de metadatos y de su forma adecuada de codificación es muy interesante para la identidad digital y el SEO (Allen, 2013; Isidoro, 2013). El conocimiento de estos metadatos se considerará imprescindible en poco tiempo no sólo para poder posicionar bien un página, sino para que un autor de blogs o de artículos publicados en la web pueda a su vez posicionarse como tal autor.

Nuestra identidad digital es un activo cada vez más importante que hay que cultivar y mantener actualizado proporcionando a los buscadores, por un lado la información que deseamos que se indexe, y por otro lado haciéndoles entender claramente que somos quienes decimos ser. En este sentido, los profesionales con dimensión social -por ejemplo, los investigadores-, pero también las empresas o las instituciones deberían ser las más interesadas en proteger y promover su identidad digital. En este contexto resulta especialmente interesante el uso de rich snippets para complementar nuestra información tanto en las redes sociales, como en las páginas de resultados de Google, de paso que mejoramos nuestro posicionamiento y en definitiva llegamos de una forma mucho más eficaz a nuestro público.

\section{1. ¿En qué consisten los rich snippets?}

Un snippet es la denominación en los buscadores de la corta descripción que muestran de cada resultado de la serp (Adrenalina, 2013; Google, 2013; Hernández, 2013). En general, cada ítem de la serp está formado por el título de la página, la descripción en sí (es decir, el snippet) y el url.

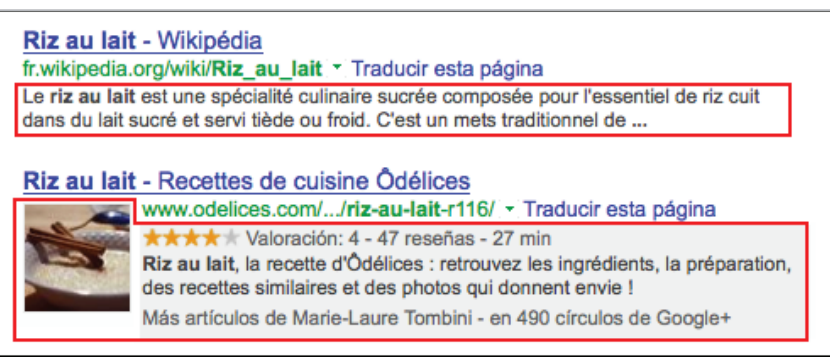

Figura 1. Comparación de snippet sin enriquecer y enriquecido

En la figura 1 podemos ver dos resultados de una búsqueda en Google sobre recetas para cocinar arroz con leche. En rojo se han marcando los snippets. En el primer caso la descripción o snippet es el tradicional, es decir no está enriquecida y aparece con una descripción muy pobre. En el segundo caso vemos un rich snippet o descripción enriquecida, y en ella se pueden ver informaciones adicionales interesantes, como la valoración de los usuarios, el tiempo de realización, el autor o la fotografía del resultado final.

Los rich snippets son descripciones enriquecidas con información adicional que será de utilidad para el usuario final. Esta información dependerá del tipo de contenido y puede ser, dependiendo de cada caso:

- nombre del autor de la página;

- fotografía de este autor;

- fecha de publicación;

- valoraciones de los usuarios; 


\section{Aclarando conceptos}

Metadatos: datos sobre los datos que normalmente incluyen información descriptiva sobre el contexto, calidad y condición o características del dato que tiene la finalidad de facilitar su recuperación, autentificación, evaluación, preservación y/o interoperabilidad (Méndez; Senso, 2004).

Microdatos: es un término con dos acepciones que genera mucha confusión.

-Significado 1: un tipo de metadatos usado, entre otras cosas, para generar los rich snippets y que se implementa con etiquetas html estándar situadas dentro del cuerpo (body) de la página web y no por un conjunto de etiquetas específicas situado en la zona del encabezamiento (head).

-Significado 2: traducción del término inglés microdata referido a un tipo específico de microdatos definido en la especificación html5.

Por tanto, el término microdatos se puede referir tanto al concepto genérico de un tipo de metadatos como a un formato concreto. Esta circunstancia suele llevar a errores y confusiones. En este artículo usaremos siempre el significado genérico de microdatos como un tipo de metadatos. Para referirnos al formato específico usaremos el término en inglés microdata. Para complicarlo todavía más, en algunas ocasiones, se produce la misma confusión de doble significado (genérico y específico) con el término microformatos (microformats). No obstante, el uso de este término es menos frecuente y generalmente se refiere al formato específico.

Rich snippets: descripciones cortas que aparecen de cada uno de los ítems de una página de resultados de un buscador y que han sido generadas por los microdatos que contienen las páginas que han originado los resultados.

Microdata, microformat y RDFa: formatos específicos de microdatos (Sporny, 2011). Todos ellos permiten generar rich snippets.

- tiempo de cocción en el caso de recetas, tal como hemos visto.

$-\ldots$

De acuerdo con la experiencia de los propios buscadores, los listados de resultados con rich snippets son más atractivos y los usuarios tienden a hacer clic más veces en los ítems donde aparecen. Por tanto los rich snippets tienen implicaciones directas en el posicionamiento web.

Los rich snippets son un claro ejemplo de la web semántica real

\section{2. ¿Qué son los microdatos?}

Son fragmentos de código realizados con html cuya función es marcar semánticamente los contenidos. No se trata de un lenguaje de etiquetado nuevo sino que usan atributos simples en etiquetas html preexistentes (en concreto, <span> $0<$ div $>$ ) para asignar nombres breves y descriptivos para indicar propiedades y elementos relativos a cada tipo de contenido.

Por ejemplo, los atributos para personas son: nombre, fotografía, afiliación...; para productos: descripción, marca, categoría...; para empresas: nombre, url, teléfono...

La forma específica en la que se utilizan las etiquetas y los atributos conduce a su vez a lenguajes o formatos distintos. Existen tres formatos básicos: microdata, microformat y RDFa. Google aconseja el uso de microdata, pero todos ellos están aceptados por los principales buscadores (Google, Yahoo y Bing), que suscribieron el acuerdo en mayo de 2009 para implementar los rich snippets en el diseño de páginas web.

Los rich snippets son un claro ejemplo de la web semántica real (Pedraza; Codina; Rovira, 2008) donde el enrique- cimiento de la web con información semántica se está produciendo de forma efectiva para la mejora de la identidad digital.

El éxito que están teniendo demuestra que las empresas relacionadas con el negocio de las búsquedas necesitan que las informaciones que se publican en la web contengan una combinación de elementos estructurados y metadatos. Ambas cosas han estado siempre en el corazón de las ciencias de la documentación.

\section{Formato de microdatos}

A continuación explicamos con más detalle qué son los microdatos indicando las características principales de los tres formatos disponibles.

\subsection{Microdata}

Están diseñados para cumplir la especificación html5 sobre microdatos. En las figuras 2 y 3 se muestra un fragmento del código de una página web antes y después de incorporar los rich snippets. Al utilizar etiquetas como <div> que no tiene ningún equivalente visual en el navegador (a menos que se desee, utilizando CSS) la apariencia para el usuario final no quedará modificada. En cambio los buscadores tendrán información adicional de tipo semántico, como por ejemplo que la cadena de caracteres "Roberto Sánchez" corresponde al nombre de una persona que trabaja en la empresa "ACME S.A."

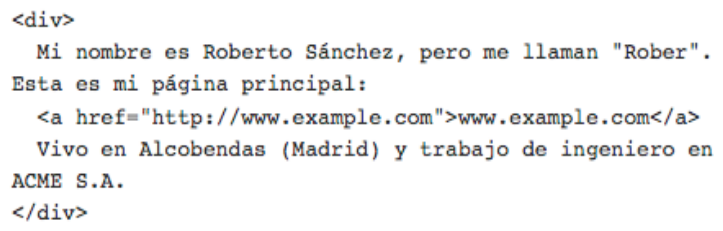

Figura 2. Código antes de incorporar microdatos 
<div itemscope itemtype="http://data-vocabulary.org

/Person" $>$

Soy <span itemprop=" name" >Roberto Sánchez</span>,

pero me llaman <span itemprop="nickname" $>$ Rober $</$ span $>$.

Esta es mi página principal:

$<a$ href="http://www.example.com"

itemprop $="$ url $">$ www .example.com $</ a>$

Vivo en Alcobendas (Madrid) y trabajo de <span

itemprop="title" $>$ ingeniero</span>

en <span itemprop="affiliation">ACME S.A. $</$ span>.

$</$ div $>$

Figura 3. Código después de incorporar microdatos en formato microdata http://support.google.com/webmasters/bin/answer.py?hl=es\&answer=176035

\subsection{Microformat}

Su característica diferenciadora es que utiliza el atributo "class", originalmente pensado para la asignación de estilos, pero actualmente aceptado para indicar información semántica. Con este formato se produce una situación curiosa puesto que una única etiqueta "class" cumple dos funciones:

- indicar cómo se presentará la información de acuerdo con una especificación de hoja de estilo (función original);

- proporcionar información semántica al buscador (rich snippet).

Los atributos "class" van igualmente asociados a etiquetas $<$ span> o < div>, igual que en los otros dos formatos, con el fin de no alterar la presentación de la información en el navegador. La class "vcard" que aparece siempre al principio del rich snippet permite identificar que se trata de un formato microformat.

$<$ div $>$

<img src="www. example.com/robertosanchez,jpg" />

<strong >Roberto Sánchez</strong>

Editor principal de Ediciones ACME

Calle Mayor

Alcalá de Henares, Madrid 28801

$</$ div $>$

Figura 4. Código antes de incorporar microdatos

$<$ div class="vcard">

<img class="photo"

src="www. example.com/robertosanchez $\cdot$ jpg" />

<strong class="fn" $>$ Roberto Sánchez $</$ strong $>$

<span class="title">Editor principal</span> de <span class="orq" $>$ Ediciones $\mathrm{ACME}</$ span $>$

<span class="adr">

<span class="street-address">Calle Mayor $4</$ span>

<span class="locality">Alcalá de Henares</span>, <span class="region" $>$ Madrid $</$ span $>$

<span class="postal-code">28801</span>

$</$ span $>$

$</$ div $>$

Figura 5. Después de incorporar microdatos en formato microformat http://support.google.com/webmasters/bin/answer.py?hl=es\&answer=146897

\section{3. $R D F a$}

Sigue las mismas características indicadas en los dos casos anteriores: uso de etiquetas <span > o < div> para incorporar información semántica sin que la visualización exterior quede modificada. Pero en este caso se usa el atributo "property" para indicar los elementos propios de este formato.

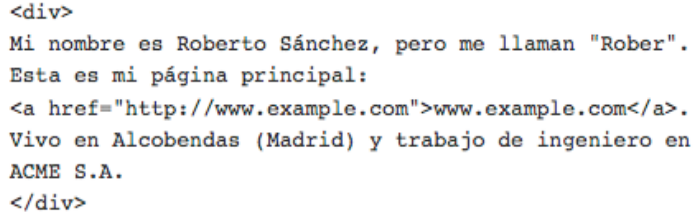

Figura 6. Código antes de incorporar microdatos

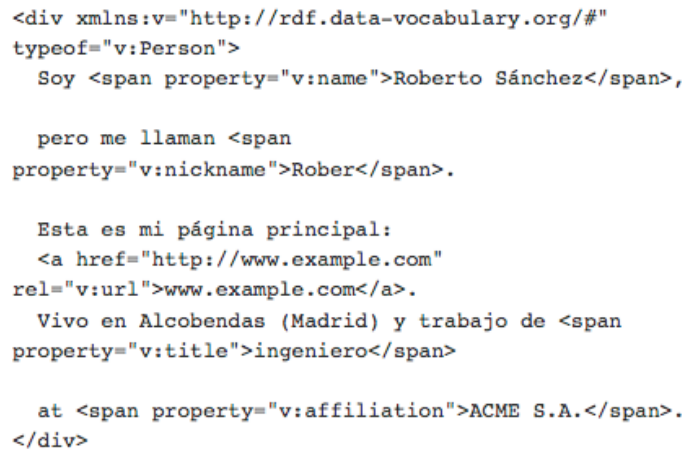

Figura 7. Código después de incorporar microdatos en formato $R D F a$ http://support.google.com/webmasters/bin/answer.py?hl=es\&answer=146898

\section{Tipos de rich snippets}

Hemos visto de qué forma se puede codificar información en el interior de una página web para que los buscadores puedan utilizarla en sus listas de resultados. Es decir, los microdatos forman el input, y los rich snippets el output.

\section{Los rich snippets son descripciones enri-} quecidas con información adicional que será de utilidad para el usuario final

Ahora vamos a ver que existen diferentes tipos de contenidos a los que se pueden aplicar los rich snippets como output. En concreto, Google reconoce en estos momentos rich snippets para:

\section{-personas}

-opiniones

-noticias

-empresas y organizaciones

-eventos

-recetas

-productos

-estructura de tabla.

Para cada tipo de contenido hay definidos unos atributos concretos que permiten indicar al buscador cuáles son las principales características de ese contenido. A continuación se presentan algunos ejemplos. 


\section{Rich snippet de persona}

\section{Matt Cutts: Gadgets, Google, and SEO -

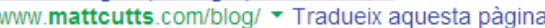 per Matt Cutts - en 256.211 cercles de Google+ Fa 5 dies - I was on your website www. google.com and wanted to shoot you quick note. I think I can make a few changes (aesthetically and/or SEO ... About Me - 30 days - PubCon 2012 slides - Out for a few days}

Figura 8. La fotografía es el elemento central de la identidad digital

Rich snippet de opinión: valoración de un usuario

\section{Rich Snippet - Destaca frente a tu Competencia}

www.adrenalina.es , ... , SEO Técnico - Tradueix aquesta pàgina

$\star \star \star \star \star \star 28$ vots

Historia y evolución de los Rich Snippet, descubre que tipos existen. El contenido enriquecido es un vocabulario de marcado entre los creadores de portales.

Figura 9. La posibilidad de otorgar votos ofrece una buena visibilidad

Rich snippet de noticia: la noticia se destaca, aun no leyendo el sitio web del medio. Para que se genere en Google es necesario dar de alta el sitio web de noticias en Google News y crear un sitemap específico de noticias de acuerdo con las indicaciones de Google.

Wert, abucheado en Sevilla, cancela una ... - El Huffington Post oll www. huffingtonpost.es/.../jose-ignacio-wert-obli... - Tradueix aquesta pàgina 14/01/2013 - El ministro fue recibido con aplausos de un sector del público y con abucheos y gritos de "Wert dimisión", "fuera, fuera" y "Universidad pública ...

Figura 10. Las primeras líneas de la noticia atraen al usuario

Rich snippet de empresas u organizaciones con ubicación por medio de Google Maps.

\section{Museo Nacional del Prado \\ www.museodelprado.es/ - \\ Página web oficial del Museo Nacional del Prado (Madrid, España) \\ Valoración: 26 / 30 - 307 reseñas de Google · Escribir una reseña \\ Calle Ruiz de Alarcón, 2328014 Madrid 913302800}

Figura 11. Además de ofrecer dirección y teléfono, el enlace indica la situación en Google maps

Rich snippet de eventos: permite posicionar eventos, conferencias, cursos, etc.

\begin{tabular}{|c|c|c|}
\hline \multicolumn{3}{|c|}{$\begin{array}{l}\text { Conciertos en Barcelona } 2013 \text { | Comprar entradas de ... - Nvivo all } \\
\text { www nvivo es/conciertos-en/Barcelona/Barcelona }\end{array}$} \\
\hline \multicolumn{3}{|c|}{$\begin{array}{l}\text { En nvivo.es puedes comprar entradas anticipadas para conciertos en Barcelona } \\
\text { Disponemos de una completísima agenda de conciertos y festivales en ... }\end{array}$} \\
\hline $\begin{array}{l}\text { dom } 23 \text { jun } \\
\text { dom } 23 \text { jun } \\
\text { dom } 23 \text { jun }\end{array}$ & $\begin{array}{l}\text { Dj Amable, Dj Gato en ... } \\
\text { Beatlemania en Barcelona } \\
\text { Joan Chamorro, Raynald ... }\end{array}$ & $\begin{array}{l}\text { Barcelona } \\
\text { Barcelona } \\
\text { Barcelona }\end{array}$ \\
\hline & & \\
\hline
\end{tabular}

Figura 12. Ofrece los datos necesarios para localizar el evento que pueda interesar

Rich snippet de receta: una web culinaria o gastronómica puede promocionar sus recetas de forma atractiva.
Arroz con verduras - El Aderezo - Blog de Recetas de Cocina www.eladerezo.com/.../arroz-con-v ... . Tradueix aquesta pàgina $\star \star \star \star \star 370$ comentaris

No sabes que hacer con esas verduras que tienes huerfanas por la nevera? Te propongo que prepares con ellas un delicioso arroz que hará las delicias de tus ...

Figura 13. Permite incluir las primeras líneas de la receta, otorgar votos y realizar comentarios

Rich snippet de producto: el usuario encuentra información específica de un producto de forma sencilla, incluyendo el precio.

\section{Nuevo Golf $<$ Modelos $<$ Volkswagen España oll}

www.volkswagen.es/es/models/golf_7.html - Tradueix aquesta pàgina ${ }^{*}$ PVP recomendado en Península y Baleares de 17.100€ para un Golf Edition BlueMotion Technology 1.6 TDI 105 CV/ 77 kW 3p. (IVA, transporte, impuesto de ...

Figura 14. Los datos más importantes para un posible comprador del automóvil

Rich snippet de productos aplicado a software (o app): útil para promocionar descargas de aplicaciones, juegos, programas, etc.

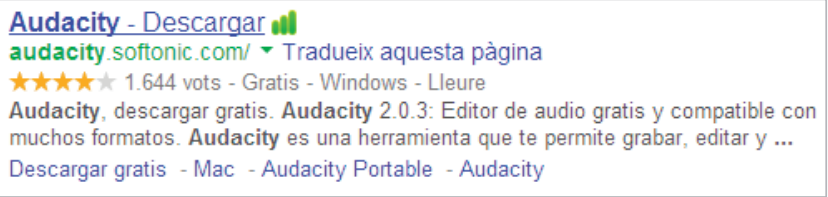

Figura 15. Datos de la versión y enlaces directos que interesan a un usuario

Rich snippet con estructura de tabla: una forma de representar diversos datos situados dentro del sitio web, ver la parte inferior con dos líneas de precio.

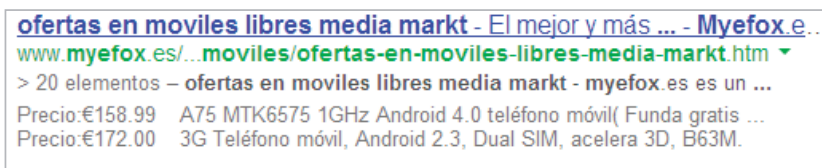

Figura 16. Las tablas permiten seleccionar los datos ofrecidos

\section{Implementación de rich snippets de personas}

Tiene una especial importancia dado el cambio en el algoritmo de búsqueda de Google de 2013. Esta última versión tiene como novedad la necesidad de identificar y mostrar la autoría de los contenidos si se desea que Google aplique el así llamado AuthorRank (Pizcos, 2013), un complemento cada vez más importante del PageRank. A continuación se describe el proceso que hay que seguir para añadir un rich snippet de persona en una página web en los listados de resultados de Google.

\subsection{Marcar el código de la página html}

El primer paso a realizar consiste en incluir los microdatos en una página web que contenga los datos de la persona. Normalmente se hará en la página o en el blog personal. 
Se pueden usar cualquiera de los 3 formatos indicados; no obstante recomendamos usar microdata debido a que es el formato preferente para Google.

Un ejemplo de código correcto sería el que se muestra en la figura 17 (en rojo las etiquetas correspondientes a los microdatos).

\section{$<$ div itemscope itemtype="http://data-vocabulary.org/Person"> \\ $<$ p $><$ span itemprop="name" $>$ Toni del Val $<$ span $>$ es $<$ span itemprop="title" $>$ profesor</span> de la <span itemprop="affiliation"> Universidad Pompeu \\ Fabra</span> desde el año 1992 en el área de Biblioteconomía y \\ Documentación. Imparte docencia en los grados de Periodismo y de \\ Comunicación Digital. $</ p><p>$ Ver \\ $<$ a href="https://plus.google.com/u/0/106790387638254532529?rel=author" > perfil en Google $+</ a\rangle\langle/ p\rangle\langle/$ div $>$}

Figura 17. Código con microdatos en formato Microdata

El código de los microdatos no se detecta externamente. Estos dos párrafos se ven en la página web como se indica en la figura 18.

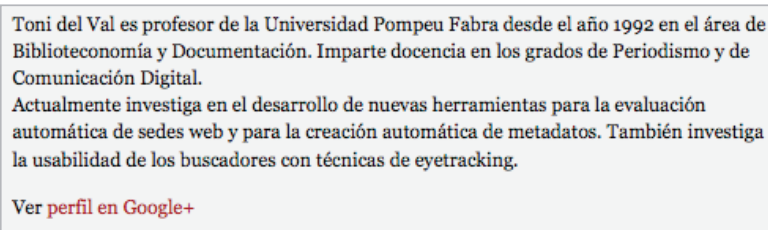

Toni del Val es profesor de la Universidad Pompeu Fabra desde el año 1992 en el área de Biblioteconomía y Documentación. Imparte docencia en los grados de Periodismo y de Comunicación Digital.

Actualmente investiga en el desarrollo de nuevas herramientas para la evaluación automática de sedes web y para la creación automática de metadatos. También investiga la usabilidad de los buscadores con técnicas de eyetracking.

Ver perfil en Google+

Figura 18. Fragmento de página web con microdatos

\subsection{Comprobar que el código es correcto}

Después de insertar los microdatos se aconseja comprobar que hayan sido correctamente codificados. Google proporciona una herramienta de validación que puede actuar sobre fragmentos de código de microdatos o sobre url de páginas que los contengan.

En la figura 19 se muestra el resultado de la comprobación de fragmento de código de microdato. En la parte inferior se pueden ver los datos estructurados que Google ha detectado.

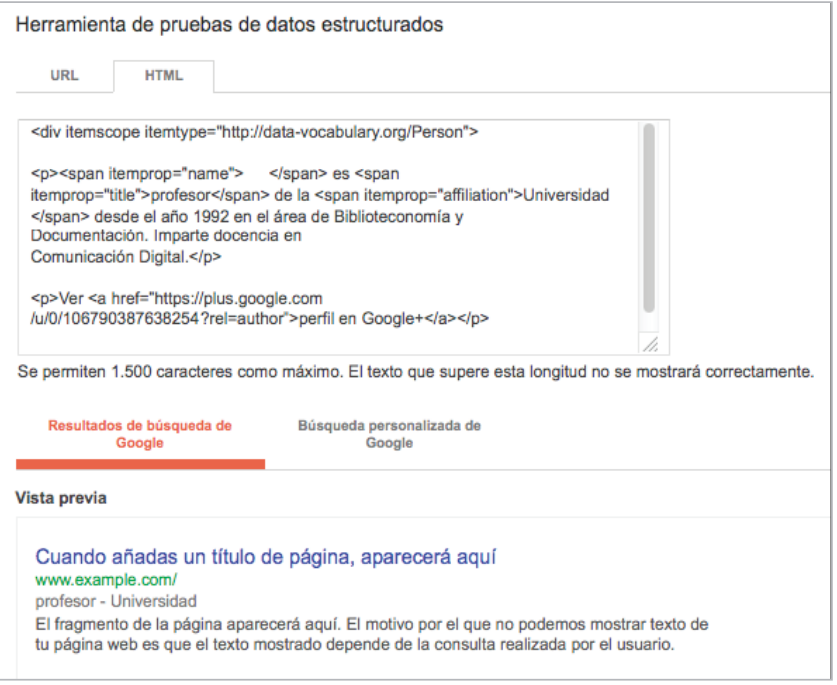

Figura 19. Herramienta de Google para validar los microdatos http://www.google.com/webmasters/tools/richsnippets

\subsection{Acreditar la autoría}

Hay que acreditar la autoría de la página web donde se han añadido los microdatos. Existen dos vías para realizar este proceso, las dos relacionadas con la posesión de un perfil en Googlet. La primera exige tener una dirección de correo electrónico con el mismo nombre de dominio que tiene el url de la página modificada. Esta condición es difícil de cumplir en muchos casos.

La segunda vía es más fácil: hay que introducir la dirección de nuestro perfil en la página web. A continuación se describe con detalle cómo hacer este proceso.

a) Dar de alta un perfil en Google+: Obviamente para iniciar el proceso hay que tener una cuenta de Google+ y procurar que nuestro perfil tenga una foto que será la que de forma automática aparecerá en el rich snippet sobre nuestra persona.

b) Poner un enlace hacia el perfil de Google+: colocar un enlace en la sección de la página web donde están los microdatos hacia la página del perfil de Google+.

c) Poner un enlace desde Google+ hacia la página: hay que poner un enlace desde el apartado "Sobre mí" + "Contribuye en" de Google+ hacia la página donde se han incluido los microdatos.

d) Volver a comprobar el código: después de acreditar la autoría se aconseja volver a usar la herramienta de validación para confirmar que Google ha detectado el doble enlace. Usando la pestaña url se verá el resultado de esta validación.

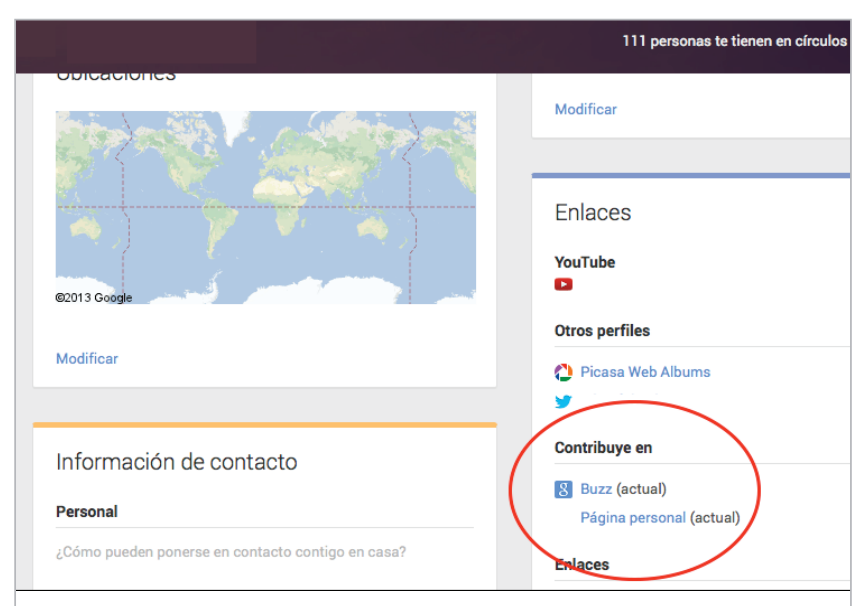

Figura 20. Nuestra página Google+ mostrando los enlaces a las dos páginas web donde a su vez hemos incluido el enlace a nuestro perfil en Google+

\subsection{Comprobación}

En el validador hemos podido ver una simulación de cómo aparecerá la fotografía en el rich snippet del listado de resultados de Google. No obstante, la aparición real de los datos tardará unos días ya que es necesaria la reindexación de los contenidos de la página modificada en los índices de Google.

Se recomienda realizar este proceso de instalación de dobles enlaces en todos los contenidos en los que seamos autores, 
especialmente en blogs. Cuando las páginas enriquecidas con microdatos y dobles enlaces aparezcan en los listados de resultados, sus descripciones estarán también enriquecidas con la fotografía del autor, ya que se mostrarán los rich snippets y no los snippets que aparecen por defecto formados a partir de un fragmento más o menos arbitrario del texto de la página.

\section{Matt Cutts: Gadgets, Google and SEO -}

uww mattcutts com/blog/ - Tradueix aquesta pàgina

per Matt Cutts - en 256.211 cercles de Google+

Fa 5 dies - I was on your website www.google.com and wanted to shoot you a quick note. I think I can make a few changes (aesthetically and/or SEO ... About Me - 30 days - PubCon 2012 slides - Out for a few days

Figura 21. Ejemplo de resultado final después de instalar con éxito el rich snippets de personas

\section{Gestores de contenidos}

Algunos gestores de contenidos analizan el código html que se incorpora usando las opciones de modificación directa del código. Como resultado de este análisis a menudo eliminan fragmentos de código con etiquetas que no corresponden a un listado relativamente reducido. Es una medida de seguridad para evitar usos fraudulentos o peligrosos. Esto ocurre especialmente con los gestores de contenidos disponibles como servicios online. Los microdatos suelen ser uno de estos fragmentos de código que de forma sorprendente desaparecen cuando los gestores analizan y modifican el código editando directamente.

Hemos podido comprobar que una de las combinaciones de gestores de contenidos y formatos de microdatos en las que el código se mantiene y por tanto que el rich snippet funciona es WordPress + formato microformats.

La figura 22 muestra un ejemplo de código que funciona bien con la versión de WordPress como servicio online (WordPress.com).

$<$ p $><$ div class="vcard" $><$ span class="fn" $>$ Toni del Val $<$ span $>$ es $<$ span class="title" $>$ profesor $<$ /span $>$ de la $<$ span class="org"> Universidad Pompeu Fabra</span> desde el año 1992 en el área de Biblioteconomía y Documentación. Imparte docencia en los grados de Periodismo y de Comunicación Digital. $</ p><p>$ Ver

$<a$ href="https://plus.google.com/u/0/106790387638254532529?rel=author" > perfil en Google+</a>

Figura 22. Código con microdato en formato microformats

Para la versión de WordPress instalada en un servidor propio (WordPress.org) hay más alternativas, como por ejemplo instalar plugins específicos para rich snippets, como "Author hReview" o "SEO Ultimate". En otros gestores de contenidos como Drupal, se puede configurar el editor para que no filtre el código html pudiendo incorporar cualquier tipo de etiqueta, incluyendo los microdatos.

\section{Directorio EXIT}

EI Directorio EXIT (Expertos en el tratamiento de la información) (Orduña-Malea; Rodríguez-Gairín; Baiget, 2007) es un servicio disponible en internet que permite a los profesionales de la archivística, la biblioteconomía, la documentación y la comunicación publicar una ficha personal con datos profe- sionales con el objetivo de fomentar la interacción entre los expertos del sector de la información y la documentación.

http://www.directorioexit.info

Las fichas de EXIT contienen algunas prestaciones avanzadas, entre las cuales y para los objetivos de este artículo cabe destacar las siguientes:

- Disponibilidad de la ficha en los formatos xml, RDF, vCard y microformats.

- Posibilidad de editar la ficha y registrar enlaces a los perfiles personales de ResearcherID, Google Citations, Mendeley, ResearchGate, Orcid y Google+.

Por tanto, el Directorio EXIT está preparado para activar los rich snippets de sus fichas cuando aparezcan los listados de resultados de Google. Tan sólo hay que realizar el proceso de acreditación de la autoría indicado en el punto 4.3 y que consiste en implementar un doble enlace:

a) Poner un enlace desde la ficha de EXIT hacia el perfil de Googlet. Para ello hay que editar la ficha de EXIT que ya está habilitada para incorporar este enlace. Tan sólo hay que escribir el identificador de Google+ que corresponde al número de 21 cifras que aparece en el url de la cuenta.

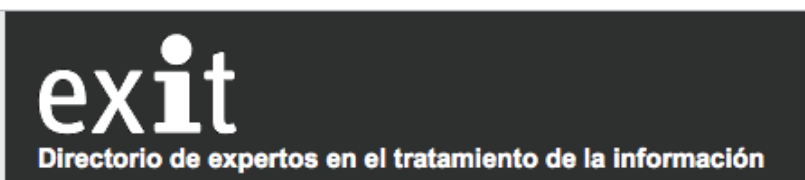

\section{Modificar}

ResearcherID:

Google+

Google Citations ID:

Mendeley ID:

ResearchGate ID:

ORCID ID:

¿Qué es?

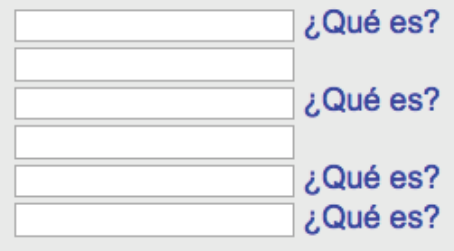

Figura 23. Modificar la ficha del Directorio EXIT

b) Dar de alta un enlace en el perfil de Google+ hacia el Directorio EXIT. Tiene que quedar situado en el apartado "Sobre mí" + "Contribuye en" del perfil de Google+.

\section{Enlaces}

\section{Contribuye en}

$$
\begin{aligned}
& 8 \text { Buzz (actual) } \\
& 8 \text { Página personal (actual) } \\
& \text { i. Directorio Exit (actual) }
\end{aligned}
$$

Figura 24. Enlace desde Google+ a la ficha del Directorio EXIT

Después de implementar estos dos enlaces se recomienda comprobar que todo funciona correctamente con la herramienta de Google para validar microdatos.

http://www.google.com/webmasters/tools/richsnippets 
La aparición del rich snippet en las búsquedas no será inmediata ya que será necesario que Google reindexe la ficha de EXIT.

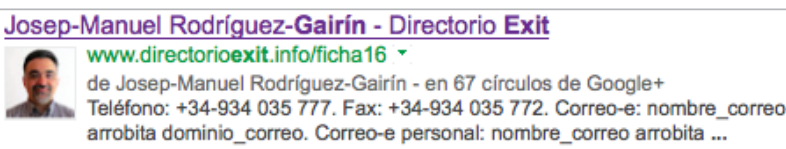

Figura 25. Rich snippet en los listados de Google generado por los microdatos del Directorio EXIT

Para los buscadores como Google la identidad digital es cada vez más importante

\section{Conclusiones}

Para los buscadores como Google la identidad digital es cada vez más importante, por un lado porque forma parte de su lucha contra el spam, y por otro porque les permite mejorar la calidad de sus resultados posicionando los mismos a partir del prestigio de los autores. Para lo cual antes tienen que estar seguros de que saben identificar quién es el autor de cada blog o de cada artículo.

Por este motivo buena parte de la ingeniería de Google para refinar su algoritmo de ordenación se basa en un doble movimiento basado por un lado en determinar cada vez de forma más certera la calidad de los contenidos y por otro de asegurar su autenticidad.

Al mismo tiempo, los buscadores buscan proponer páginas de resultados cada vez más útiles para los usuarios incorporando más información semántica, estructurada y detallada, pero sin tener que recurrir a un procesamiento manual. En este escenario se unen los intereses de los buscadores para ofrecer resultados de mayor calidad y de los profesionales y empresas que crean contenidos originales. El punto de unión de estos intereses está en el uso adecuado de metadatos y de un formato de codificación que los buscadores puedan interpretar, y éste es el papel de los microdatos, siendo los rich snippets uno de sus productos.

En este artículo hemos podido ver las posibilidades que nos ofrecen los rich snippets para mejorar la presentación de nuestras páginas en los listados de resultados de Google usando los microdatos. Se ha incidido especialmente en los rich snippets de personas ya que tienen una influencia directa en la mejora de la presencia personal en la Red y en la reputación online a través del concepto cada vez más importante para Google del AuthorRank.

\section{Nota}

Artículo financiado en parte por los proyectos: Comunicación online de los destinos turísticos CSO 2011-22691 y Audiencias activas y periodismo CSO2012-39518-C04-02. Plan Nacional de I+D+i, Ministerio de Economía y Competitividad (España).

\section{Bibliografía}

Adrenalina (2013). "Rich snippet - Destaca frente a tu competencia". Blog Adrenalina.

http://www.adrenalina.es/rich-snippet

Allen, Jack (2013). "Hacking authorship for SEO: advantages and gaining insight". Search engine journal, May 3.

http://www.searchenginejournal.com/hacking-authorshipfor-seo-advantages-and-gaining-insight/62284

Google (2013). "About rich snippets and structured data. Rich snippets (microdata, microformats, RDFa, and data highlighter)". Google webmaster tools, June 5.

http://support.google.com/webmasters/bin/answer.py?hl= en\&answer $=99170 \&$ topic $=1088472 \&$ ctx $=$ topic

Hernández, Chris (2013). "Poner la cara del autor en resultados de Google iy que funcione!". Activa la web, abril 18. http://www.activalaweb.com/poner-mi-cara-enresultados-de-google

Isidoro, Andrew (2013). "Increase distribution for your branded content with microformats". Content Marketing Institute, May 3.

http://contentmarketinginstitute.com/2013/05/increasedistribution-branded-content-microformats

Méndez, Eva; Senso, José-Antonio (2004). Introducción a los metadatos: estándares y aplicación. Unidad de autoformación, Sedic.

http://www.sedic.es/autoformacion/metadatos/index.htm

Orduña-Malea, Enrique; Rodríguez-Gairín, Josep-Manuel; Baiget, Tomàs (2007). "Directorio de expertos en el tratamiento de la información (EXIT). Análisis de uso". El profesional de la información, v. 16, n. 5, pp. 497-509.

http://www.elprofesionaldelainformacion.com/contenidos/2007/ septiembre/13.pdf

http://dx.doi.org/10.3145/epi.2007.sep.13

Pedraza, Rafael; Codina, Lluís; Rovira, Cristòfol (2008). "Semantic web adoption: online tools for web evaluation and metadata extraction". En: Da-Ruan et al. (ed) Computational intelligence in decision and control. Procs of the $8^{\text {th }}$ Int Flins conf. New Jersey: World Scientific Publishing Co Pte Ltd, 2008. http://repositori.upf.edu/handle/10230/16353

Pizcos, Carlos (2013). "El author rank ya es un factor de posicionamiento". Pizcos, 16 mayo. http://www.pizcos.net/2013/05/author-rank-factor.html

Sporny, Manu (2011). "An uber-comparison of RDFa, microdata and microformats". The beautiful, tormented machine, June 25. http://manu.sporny.org/2011/uber-comparison-rdfa-md-uf 